\title{
Why Can't I Measure the External Quantum Efficiency of the Ge Subcell of my Multijunction Solar Cell?
}

\author{
Enrique Barrigón ，Pilar Espinet-González , Yedileth Contreras \\ and Ignacio Rey-Stolle
}

\begin{abstract}
The measurement of the external quantum efficiency (EQE) of low bandgap subcells in a multijunction solar cell can be sometimes problematic. In particular, this paper describes a set of cases where the EQE of a Ge subcell in a conventional GaInP/GaInAs/Ge triple-junction solar cell cannot be fully measured. We describe the way to identify each case by tracing the I-V curve under the same light-bias conditions applied for the EQE measurement, together with the strategies that could be implemented to attain the best possible measurement of the EQE of the Ge subcell.
\end{abstract}

\section{INTRODUCTION}

Nowadays, GaInP/GaInAs/Ge triple-junction solar cells (3JSC) show efficiencies close to $42 \%$ [1]. Despite these outstanding results, the characterization of these devices is not straightforward. In particular, many laboratories as well as solar cell and CPV-module manufacturers experience difficulties when trying to measure the external quantum efficiency (EQE) of the Ge subcell in a multijunction solar cell (MJSC). Either it is not possible to obtain any response, or the so-called measurement artifact appears. This artifact consists of a lower than expected EQE of the Ge subcell under test together with the simultaneous measurement of some response in wavelengths corresponding to another subcell. The measurement artifact has been related to the presence of a low shunt resistance in the Ge junction [2], luminescence coupling (LC) [3] or a low breakdown voltage $\left(\mathrm{V}_{\mathrm{br}}\right)$ in conjunction with an incorrect voltage bias $\left(\mathrm{V}_{\text {bias }}\right)[4]$.

In this paper, we will present cases studies representative for several limit cases when no signal can be measured or a low signal is obtained instead from the Ge subcell. In particular, we will analyze the following cases where:

1. The Ge subcell shows a very low shunt resistance.

2. The Ge subcell shows an extremely low $\mathrm{V}_{\mathrm{br}}$.

3. The presence of LC between subcells alters the effective light bias condition of the BC.

\section{EXPERIMENTAL DETAILS}

The setup used for the EQE measurements consists of a Xe lamp used as white light source which passes through a Horiba Jobin Yvon monochromator (TRIAX180) and a filter wheel. The monochromatic light is chopped at a frequency of $481 \mathrm{~Hz}$ and optically guided to the device under test and to a monitor cell. Two different low noise current preamplifiers (SR570) convert the output currents into voltages from the device under test (DUT) and the monitor cell, respectively-, which are then measured by a two-channel lock-in amplifier (Anfatec 203). The monitor cell is always measured and corrects the lamp fluctuations that might take place during the measurement. A set of lasers $(632.8 \mathrm{~nm}$ and $806 \mathrm{~nm}$ ) and a halogen lamp with different optical filters constitute the multi-source spectrally tunable light bias used along the study. The low noise current preamplifier is also used to voltage bias the device under test. 
In principle, in order to measure the $\mathrm{EQE}$ of a Ge subcell in a MJSC configuration, this subcell should be subilluminated (as compared to to the other subcells in the stack), though it still should generate the appropriate one-sun photocurrent. Throughout the text, the expression "X-limiting light bias" will be employed to refer to the special combination of light sources needed for the EQE measurement of the X subcell, where X might be the top (TC), middle (MC) or bottom subcell (BC). As explained in Ref. [5], the I-V curve of the MJSC -measured with a sourcemeter instrument Keithley 2602-, taken under the same light bias conditions to be applied for the EQE measurement, will be employed to assess the problems that might be affecting the $\mathrm{EQE}$ measurement.

All the MJSC measured in this work are lattice-matched GaInP/GaInAs/Ge 3JSC. Some of them are commercial solar cells, whereas other have been grown and manufactured at our facilities [6]. Each of the cases shown in this paper corresponds to a different and particular solar cell.

\section{RESULTS AND DISCUSSION}

\section{Ideal Case}

Let us first consider the ideal case, where the EQE of the Ge subcell in a 3JSC can be measured following the standard procedure. As can be seen in Fig.1(a), after applying a BC-limiting light bias, the $\mathrm{EQE}$ of the Ge $\mathrm{BC}$ is measured. Furthermore, there is no difference in the signal if we sweep the $\mathrm{V}_{\text {bias }}$ applied over a $1.5 \mathrm{~V}$ range. This indicates that the solar cell shows neither shunt resistance problems nor a low breakdown voltage, as it will be shown below.

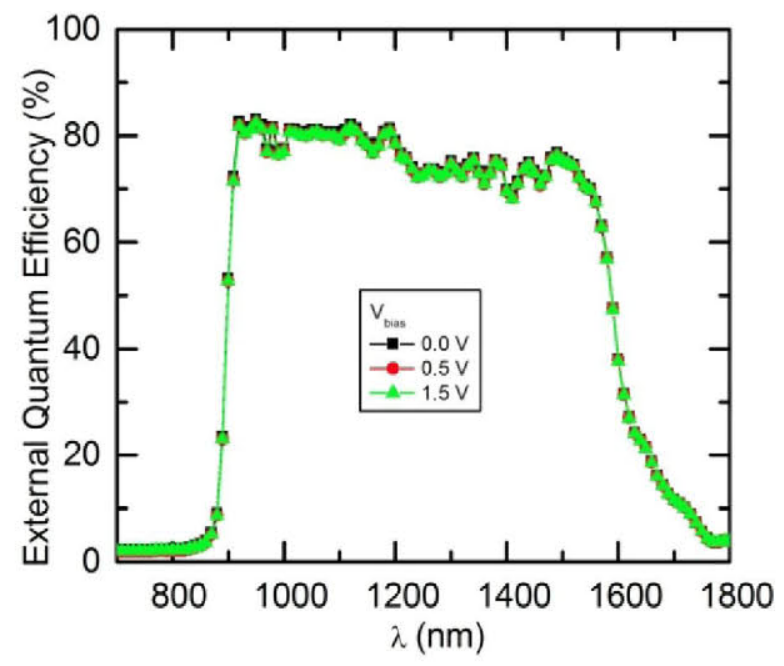

FIGURE 1. Ideal case of a EQE measurement of a Ge BC under different $\mathrm{V}_{\text {bias }}$ condition.

\section{Low Shunt Resistance Problem}

Figure 2(a) shows the EQE signal measured after applying a BC-limiting light bias and a $\mathrm{V}_{\text {bias }}$ of $2.00 \mathrm{~V}$ (according to the standard $\mathrm{V}_{\text {bias }} \approx \Sigma V_{o c}^{\text {non-limiting }}$ ). The wavelength range shown in the figure is from 500 to 1100 $\mathrm{nm}$ in order to cover at the same time (and with some detail) the wavelengths corresponding to the EQE of the TC ( $500-700 \mathrm{~nm}), \mathrm{MC}(\sim 500-950 \mathrm{~nm})$ and BC (from $900 \mathrm{~nm}$ on). As can be seen in the figure, instead of having a signal corresponding to the $\mathrm{Ge} \mathrm{BC}$, the $\mathrm{EQE}$ shows a convolution of the $\mathrm{TC}, \mathrm{MC}$ and $\mathrm{BC} \mathrm{EQEs}$. The figure also shows the measured signal when $\mathrm{V}_{\text {bias }}$ has been swept from $0.5 \mathrm{~V}$ to $2.0 \mathrm{~V}$. As can be observed, no matter the value of $\mathrm{V}_{\text {bias }}$ applied in this range, the $\mathrm{EQE}$ of the $\mathrm{BC}$ cannot be measured. The problem persists when applying higher light bias on the non-limiting subcells (not shown in the figure). Only after the I-V curve is traced (see Fig.2(b)), it is found that the Ge subcell shows indeed an evident case of a low shunt resistance problem, which makes it impossible to measure the $\mathrm{EQE}$ of the Ge subcell. It is interesting to note that such $\mathrm{R}_{\text {shunt }}$ problem is only affecting the Ge subcell. In fact, for $\mathrm{V}_{\text {bias }}$ lower than $-0.25 \mathrm{~V}$ the current shows a constant value which indicates that instead of 
the $\mathrm{Ge} \mathrm{BC}$, another subcell starts current-limiting the device (the TC, in this example).Besides, it can be observed that the TC, for this particular 3JSC, shows a high shunt resistance value, unlike the BC.

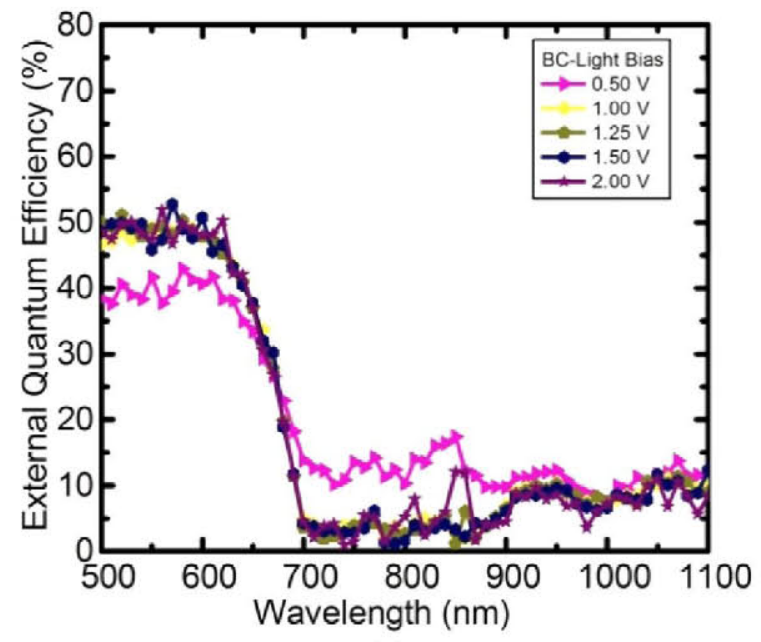

(a)

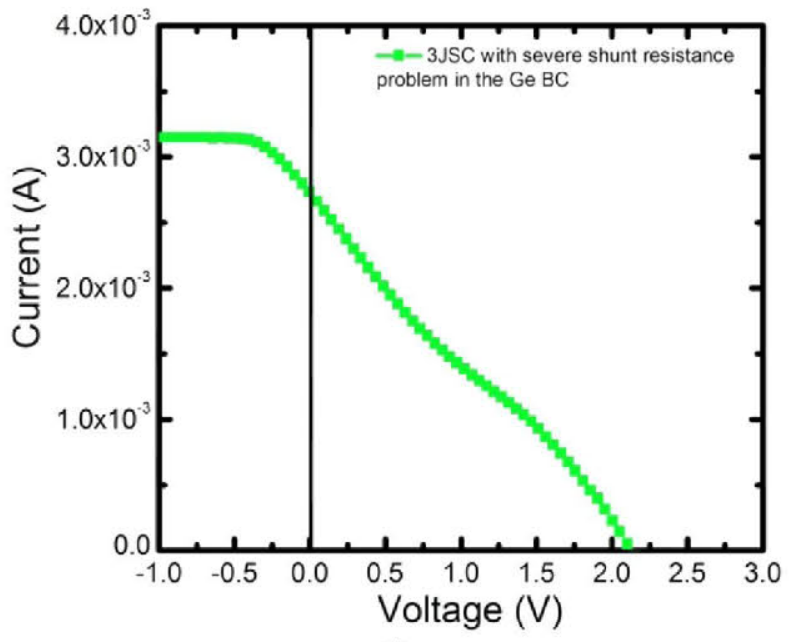

(b)

FIGURE 2. (a) EQE of a Ge BC under different $V_{\text {bias }}$ for the case of a low shunt resistance. (b) I-V curve of the 3 JSC obtained under the same light-bias conditions applied as in (a)..

\section{Low $V_{b r}$ of the Ge Subcell}

In the presence of a $\mathrm{BC}$ with a low breakdown voltage, the I-V curve of the MJSC under BC-limiting light bias conditions shows a characteristic shape, as the black curve shown in Fig.3 [7]. Under this particular light bias, the $\mathrm{BC}$ produces much less current than the middle cell, which in turn is slightly less excited than the top cell $\left(\mathrm{I}_{\mathrm{BC}}<\mathrm{I}_{\mathrm{MC}}<\mathrm{I}_{\mathrm{TC}}\right.$ ). If the $3 \mathrm{JSC}$ is voltage-biased at short-circuit, then the $\mathrm{I}_{\mathrm{sc}}$ measured actually corresponds to the second limiting junction (i.e., the $\mathrm{MC}$ ) since the $\mathrm{Ge} \mathrm{BC}$ is in breakdown with a current higher than the photogenerated current in the MC. As soon as a voltage higher than $1.75 \mathrm{~V}$ is applied to the $3 \mathrm{JSC}$, the current in the I-V curve starts to decrease (i.e., the $\mathrm{BC}$ is progressively leaving the breakdown state) until a constant value is achieved, which actually corresponds to that of the photogenerated current in the $\mathrm{BC}$.

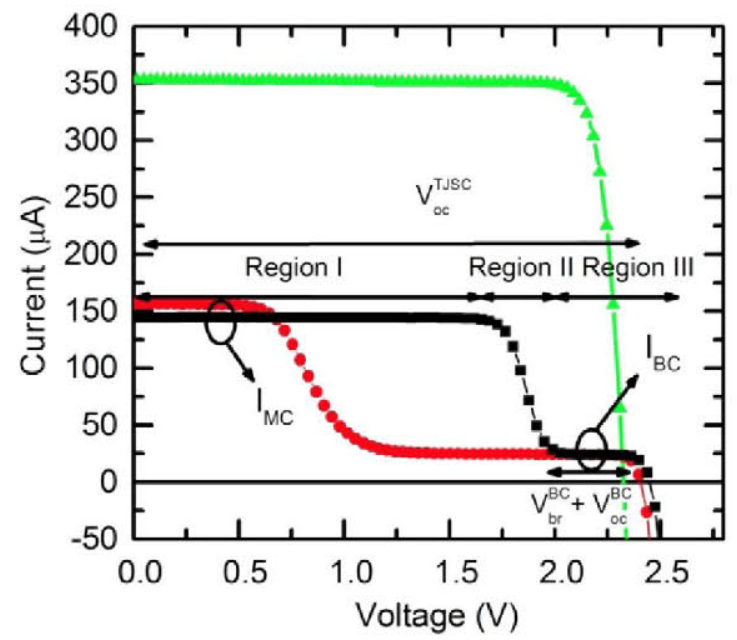

FIGURE 3. I-V curves under a BC-limiting light bias for three different $3 \mathrm{JSC}$ with a low $\mathrm{V}_{\mathrm{br}}$ in the BC. 
The shape of such I-V curve has many implications from the point of view of the EQE measurement. Firstly, the EQE measured at $0.00 \mathrm{~V}$ will correspond to the second limiting subcell (in our experiment the MC), as can be seen in Fig.4(a). In order to measure the EQE of the $\mathrm{Ge} B C$, an external $\mathrm{V}_{\text {bias }}$ must be applied. Under a $\mathrm{V}_{\text {bias }}$ of $2.20 \mathrm{~V}$, the $\mathrm{EQE}$ of the Ge $\mathrm{BC}$ is effectively measured, as seen also in Fig.4(a). In order to understand the dependence of the $\mathrm{EQE}$ versus $\mathrm{V}_{\text {bias }}$, Fig.4(b) plots the EQE at 750 and $950 \mathrm{~nm}$-i.e., wavelengths where the $\mathrm{MC}$ and $\mathrm{BC}$ should show response, respectively- versus voltage bias. Whereas the $\mathrm{MC}$ is measured for $\mathrm{V}_{\text {bias }}$ lower than $1.50 \mathrm{~V}$, to measure the $\mathrm{EQE}$ of the $\mathrm{BC} \mathrm{a} \mathrm{V}_{\text {bias }}$ higher than 2.00 has to be applied. If a voltage between these two values is used, the outcome will be a composite signal mixing the $\mathrm{MC}$ and the $\mathrm{BC}$ responses, namely, a measurement artifact. Therefore, there is a clear correlation between Fig.3 and Fig.4(b). Accordingly, we can define three different regions in both curves (as indicated in both plots). On the one hand, Region I will be the region where the EQE of another subcell (different to the BC) will be measured, despite we are applying BC-limiting light bias. On the other hand, Region III is the region where the subcell under test is appropriately measured (i.e. the BC). Finally, Region II is the region where the signal is a composite of both subcells, namely, the so called artifact region. The existence of these three regions highlights the usefulness of tracing the I-V curve under a certain light bias (i.e. Fig. 3 ) to determine the suitable voltage biasing range to perform an EQE measurement.

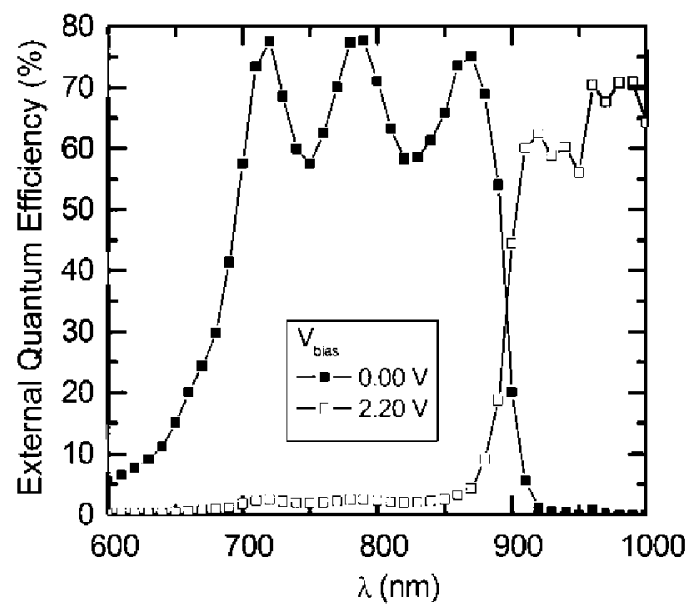

(a)

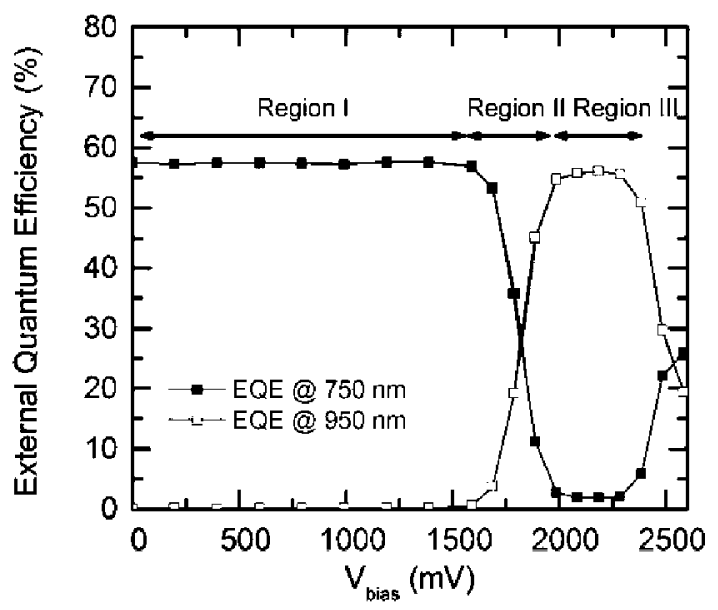

(b)

FIGURE 4. (a) EQE obtained at 0.00 and $2.20 \mathrm{~V}$ under a BC-limiting light bias for a 3JSC with a low $\mathrm{V}_{\mathrm{br}}$ in the Ge subcell (b) Evolution of the EQE at 750 and $950 \mathrm{~nm}$ with the external voltage bias.

The width of Region III mainly depends on the values of $\mathrm{V}_{\mathrm{br}}$ and $\mathrm{V}_{\mathrm{oc}}$ of the $\mathrm{BC}$ (measured under such light bias) [4]. At the same time, the $\mathrm{V}_{\mathrm{br}}$ depends on $1 / \mathrm{N}_{\mathrm{A}}$, being $\mathrm{N}_{\mathrm{A}}$ the doping level of the base layer of the $\mathrm{BC}$ [8]. Therefore, the width of Region III might change with the doping level of the Ge wafer employed since it constitutes the base of the Ge subcell. Additionally, Region II would be displaced to another voltage range. As an example, several I-V curves are also plotted in Fig. 3 corresponding to similar $3 \mathrm{JSC}$ as the one just described, but grown on Ge wafers with different doping levels. The red curve corresponds to the case of a wafer with a lower doping level, which extends the voltage range of Region III (i.e., where the $\mathrm{EQE}$ of the $\mathrm{Ge} \mathrm{BC}$ could be measured), as expected. In this case, the measurement artifact (i.e., Region II) will take place for voltages from 0.6 to $1.0 \mathrm{~V}$. On the contrary, an extreme case is represented with the green I-V curve, which corresponds to a $3 \mathrm{JSC}$ grown on a Ge wafer with $\mathrm{N}_{\mathrm{A}}>1 \cdot 10^{18} \mathrm{~cm}^{-3}$. In this case, the EQE of the Ge subcell cannot be measured since Region III has virtually disappeared. As can be seen in Fig.5(a), the EQE obtained at $0.00 \mathrm{~V}$ corresponds to the MC, as expected, while the $\mathrm{EQE}$ at $2.20 \mathrm{~V}$ shows a measurement artifact. Again, Fig.5(b) shows the EQE at 750 and $950 \mathrm{~nm}$ as in Fig.4(b), to check if there is a voltage range where the Ge BC could be measured. Only at voltages higher than $2.00 \mathrm{~V}$ there is some response of the $\mathrm{BC}$, but always accompanied with a response in the MC. In other words, due to the extremely low $\mathrm{V}_{\mathrm{br}}$ of the $\mathrm{BC}$, together with the low $\mathrm{V}_{\mathrm{oc}}$ under such light-bias conditions, there is no flat region in the I-V curve where the EQE measurement could take place. The only way to measure the EQE of this subcell would be via an isotype Germanium subcell. 


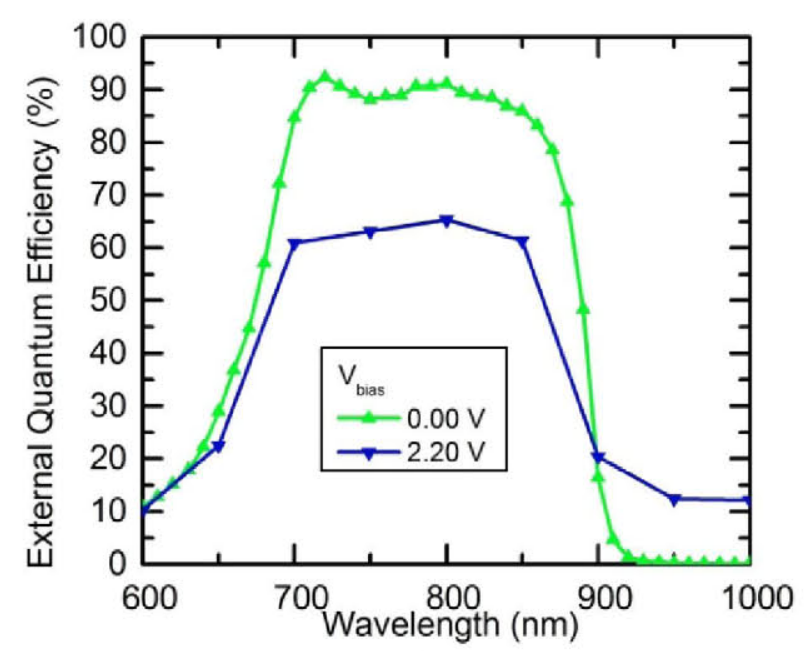

(a)

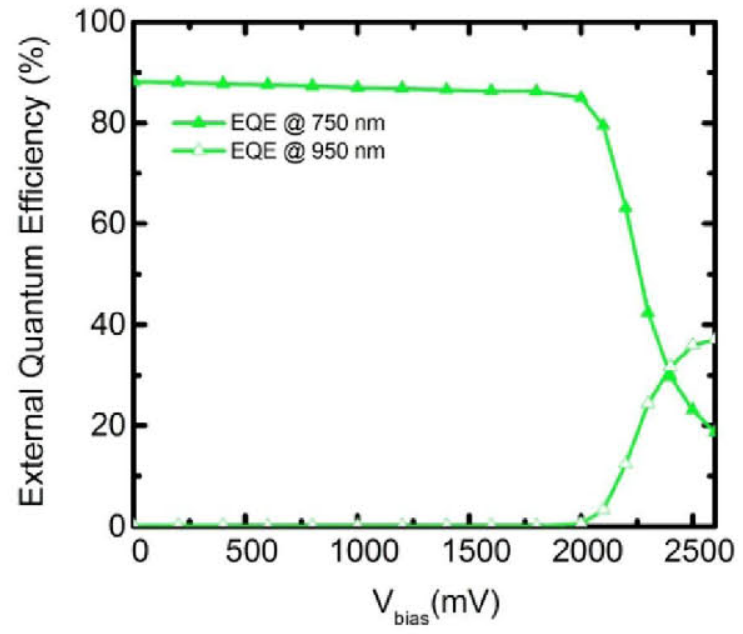

(b)

FIGURE 5. (a) EQE obtained at 0.00 and $2.20 \mathrm{~V}$ under a BC-limiting light bias for a 3JSC with an extremely low $\mathrm{V}_{\mathrm{br}}$ in the Ge subcell (b) Evolution of the EQE at 750 and $950 \mathrm{~nm}$ with the external voltage bias.

\section{Luminescent Coupling Effects}

Figure 6 shows the I-V curves of the 3JSC under several BC-limiting light bias conditions. In this case the light bias initially set makes the $\mathrm{BC}$ limit, being the $\mathrm{TC}$ the second limiting subcell ( $\mathrm{I}_{\mathrm{BC}}<\mathrm{I}_{\mathrm{TC}}<\mathrm{I}_{\mathrm{MC}}$ ). Under these conditions the black I-V curve is measured. Then the illumination of the middle cell (the most overexcited subcell) is further increased by boosting the intensity of the $806 \mathrm{~nm}$ laser and curves red, green, blue, cyan and magenta are obtained. First thing to be noted is that a stepped I-V curve -as in Fig. 3-is observed, indicating the presence of a low $\mathrm{V}_{\mathrm{br}}$. In this case, as a result of the particular configuration of the light bias used $\left(\mathrm{I}_{\mathrm{BC}}<\mathrm{I}_{\mathrm{TC}}<\mathrm{I}_{\mathrm{MC}}\right.$ ), the $\mathrm{I}_{\mathrm{sc}}$ measured at low voltage bias belongs to the TC. However, the key phenomenon observed here is that as the light bias to the MC is increased (still maintaining the $\mathrm{BC}$-limiting light bias condition), the current of the $\mathrm{BC}$ (current in Region III) also increases. This fact is indicative that the current of the $\mathrm{BC}$ is generated by two sources: the external light bias applied to the $\mathrm{Ge} \mathrm{BC}$ plus a contribution coming from the luminescent coupling from the $\mathrm{MC}$ to the $\mathrm{BC}$. This situation holds until a certain limit -and accordingly the $\mathrm{EQE}$ of the $\mathrm{BC}$ can be measured-, when the current of the $\mathrm{BC}$ is higher than that of the TC thanks to the contribution of the LC term. The magenta curve in Fig.6 represents this situation, where the I-V curve is then the result of an effective TC-limiting light bias condition. Under this circumstance, the $\mathrm{EQE}$ of the $\mathrm{TC}$ is the one to be measured despite an apparently $\mathrm{BC}$-light bias condition has been used. In order to circumvent this issue, the light bias of the $\mathrm{MC}$ should be decreased, or alternatively, the light bias of the TC should be increased. In other words, if under an external BC-limiting light bias condition, the EQE of another subcell is measured instead, then the effective light bias being received by the $\mathrm{BC}$ might be influenced by luminescent coupling effects. This situation is easily identified in the I-V curve if the current attributed to the BC does not vary upon a slight change of the $\mathrm{BC}$ light bias. 


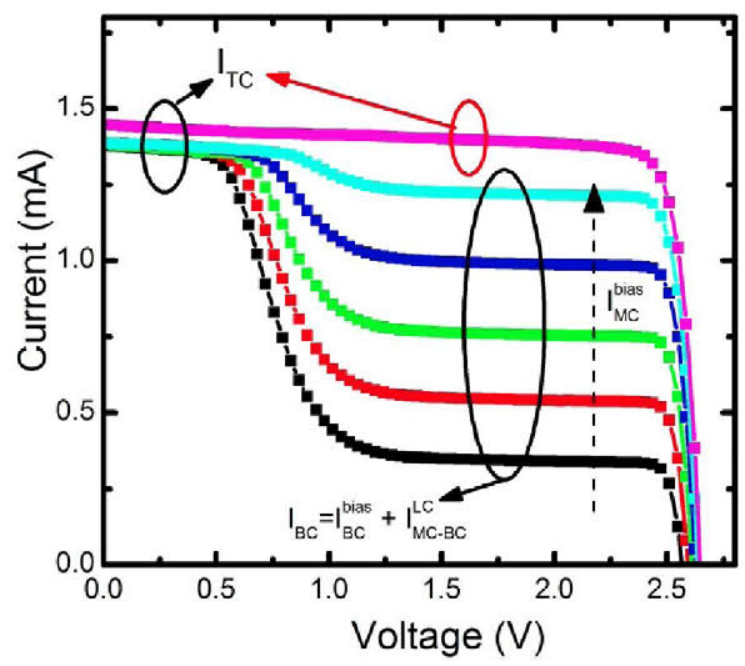

FIGURE 6. I-V curves under several BC-limiting light bias condition for $3 \mathrm{JSC}$ s with a low $\mathrm{V}_{\mathrm{br}}$ in the BC.

\section{SUMMARY AND CONCLUSIONS}

In some cases, the EQE of a Ge subcell in a conventional GaInP/Ga(In)As/Ge 3JSC cannot be measured, or a low response is obtained. Three possible origins for this problem have been identified and analyzed, namely, 1)low shunt resistance; 2) low $\mathrm{V}_{\mathrm{br}}$ of the Ge subcell; and 3) LC effects altering the effective light bias on the Ge subcell. Tracing the I-V curve under the same light bias conditions to be applied for the EQE helps determine the origin of the problems affecting the EQE measurement. Despite these cases have been studied on germanium subcells, they will generally apply for low bandgap subcells in any MJSC.

\section{ACKNOWLEDGMENTS}

Financial support from the EU Commission (LONGESST project no.607153) and from Spanish MINECO (TEC2011-28639-C02- 01, TEC2012-3728, RTC140910B01) are gratefully acknowledged. The authors would like to thank J. Bautista for his continuous support.

\section{REFERENCES}

1. King R, Boca A, Hong W, Liu XQ, Bhusari D, Larrabee D, et al. "Band-Gap-Engineered Architectures for High-Efficiency Multijunction Concentrator Solar Cells." in 24th European Photovoltaic Solar Energy Conference. Hamburg, Germany; 2009.

2. Meusel M, Baur C, Letay G, Bett AW, Warta W, Fernandez E., Prog Photovolt. 11, 499-514 (2003)

3. Lim SH, Li J, Steenbergen EH, Zhang YH., Prog Photovolt. 21, 344-350 (2013)

4. Barrigón E, Espinet-González P, Contreras Y, Rey-Stolle I. Prog Photovolt. (in press) (2015) doi: 10.1002/pip.2597

5. Barrigon E, Espinet-Gonzalez P, Contreras Y, Barrutia L, Rey-Stolle I, Algora C. "On the use of I-V curves as a diagnosis tool for proper external quantum efficiency measurements of multijunction solar cells." in 40th IEEE Photovoltaic Specialists Conference 2014. pp. 3453-6.

6. Barrigón E, Rey-Stolle I, Galiana B, Garcia I, Algora C. "GaInP/GaInAs/Ge triple junction solar cells for ultra high concentration." in Proc Spanish Conference on Electron Devices 2009 pp. 383-6

7. King DL, Hansen BR, Moore JM, Aiken DJ. "New methods for measuring performance of monolithic multijunction solar cells" in Conference Record of the Twenty-Eighth IEEE Photovoltaic Specialists Conference 2000 pp. 1197-201

8. Neudeck GW. The p-n junction diode, edited by Prentice Hall (1989). 